\title{
Estratégias promotoras de cuidado à saúde mental diante a Covid-19
}

\author{
Strategies promoting mental health care in front of Covid-19 \\ Estrategias para promover la atención de la salud mental frente al Covid-19
}

\author{
Dina Mara Formiga da Silva \\ ORCID: https://orcid.org/0000-0002-5387-7194 \\ Universidade Estadual do Ceará, Brasil \\ E-mail: dinamara_silva@hotmail.com \\ Débora Brenda Carneiro de Souza \\ ORCID: https://orcid.org/0000-0002-8503-4052 \\ Universidade Estadual do Ceará, Brasil \\ E-mail: deborabrenda@outlook.com \\ Levy Sombra de Oliveira Barcelos \\ ORCID: https://orcid.org/0000-0001-5439-4066 \\ Universidade Estadual do Ceará, Brasil \\ E-mail: levysombraodontologia@gmail.com \\ Maria Salete Bessa Jorge \\ ORCID: https://orcid.org/0000-0001-6461-3015 \\ Universidade Estadual do Ceará, Brasil \\ E-mail: maria.salete.jorge@gmail.com
}

\begin{abstract}
Resumo
Objetivo: analisar as estratégias promotoras de saúde mental durante a pandemia pela Covid-19. Metodologia: Revisão Integrativa da literatura, que tem como base as seis fases estabelecidas por Mendes, Silveira e Galvão, sendo utilizada a estratégia PCC para a formulação da pergunta norteadora, e busca detalhada e individual realizada na Pubmed, e Literatura Latino-Americana e do Caribe em Ciências da Saúde (LILACS) via Biblioteca Virtual em Saúde (BVS) em agosto de 2021. Resultados: Foram selecionados 10 artigos para análise e elaboração da matriz de síntese. Discussão: diante dos achados extraídos dos estudos selecionados, percebe-se que é fundamental levar em consideração o contexto social que a pessoa está inserida para que seja possível identificar os fatores e assim serem criadas estratégias de enfrentamento. Conclusão: este estudo permitiu refletir sobre as estratégias promotoras de saúde mental, e como elas vem sendo implementadas diante à emergência de saúde pública por conta do novo coronavírus. Palavras-chave: Covid-19; Estratégias de saúde mental; Pandemia; Saúde mental.
\end{abstract}

\begin{abstract}
Objective: to analyze the strategies promoting mental health during the pandemic by Covid-19. Methodology: Integrative literature review, which is based on the six phases established by Mendes, Silveira and Galvão, using the PCC strategy for the formulation of the northern question, and detailed and individual search conducted at Pubmed, and Latin American and Caribbean Literature on Health Sciences (LILACS) via the Virtual Health Library (VHL) in August 2021. Results: 10 articles were selected for analysis and elaboration of the synthesis matrix. Discussion: in view of the findings extracted from the selected studies, it is perceived that it is essential to take into account the social context that the person is inserted in order to identify the factors and thus be created coping strategies. Conclusion: this study allowed us to reflect on the strategies that promoting mental health, and how they have been implemented in the face of public health emergency due to the new coronavirus.
\end{abstract}

Keywords: Covid-19; Mental health strategies; Pandemic; Mental health.

\section{Resumen}

Objetivo: analizar las estrategias de promoción de la salud mental durante la pandemia por Covid-19. Metodología: Revisión integradora de la literatura, que se basa en las seis fases establecidas por Mendes, Silveira y Galvão, utilizando la estrategia del PCC para la formulación de la pregunta del norte, y la búsqueda detallada e individual realizada en Pubmed, y Literatura Latinoamericana y del Caribe en Ciencias de la Salud (LILACS) a través de la Biblioteca Virtual en Salud (BVS) en agosto de 2021. Resultados: Se seleccionaron 10 artículos para el análisis y elaboración de la matriz de síntesis. Discusión: en vista de los hallazgos extraídos de los estudios seleccionados, se percibe que es esencial tener en cuenta el contexto social que la persona está insertada para identificar los factores y así crear estrategias de afrontamiento. Conclusión: este estudio nos permitió reflexionar sobre las estrategias que promueven la salud mental, y cómo se han implementado ante la emergencia de salud pública por el nuevo coronavirus.

Palabras clave: Covid-19; Estrategias de salud mental; Pandemia; Salud mental. 


\section{Introdução}

A pandemia da Covid-19 se caracteriza como uma infecção respiratória provocada pelo Coronavírus da Síndrome Respiratória Aguda Grave 2 (SARS-CoV-2), vírus altamente patogênicos (Ministério da Saúde, 2020a). É descrita como um dos maiores problemas de saúde pública internacional das últimas décadas e tem afetado a população tanto no que concerne ao adoecimento físico, como mental.

No Brasil, foi decretado pelo Governo Federal, por meio da portaria $\mathrm{n}^{\circ} 340$, de 30 de março de 2020 , medidas de Distanciamento Social, visando a necessidade dos indivíduos com suspeita do vírus e/ou sintomáticos de manter-se em isolamento, como meio de diminuir a progressão e disseminação do vírus, resultando em controle, e menores taxas de morbidade e mortalidade (Ministério da Cidadania, 2020).

Ainda nesse cenário foi decretado em vários países, inclusive no Brasil, medidas determinando o fechamento de universidades, escolas, edifícios de escritórios, shoppings, comércios em geral, entre outros. Com isso, o sentimento de perda do direito de ir e vir, pode levar a uma negação da severidade da pandemia, e consequentemente a desvalorização da importância do Distanciamento Social através de atitudes e comportamentos diante o problema. A população italiana em geral relatou uma alta prevalência de problemas de Saúde Mental durante as últimas semanas de bloqueio (Gualano, Lo Moro, Voglino, Bert \& Siliquini, 2020).

Com as imposições de lockdown, quarentena, que consistiu em distanciar e restringir a movimentação de indivíduos já expostos a situações com potencial de contágio; e o isolamento, em afastar os infectados ou aqueles que apresentaram sintomas característicos da doença dos indivíduos que estavam sadios, medidas que foram impostas para diminuir a disseminação do vírus, acabaram impactando na saúde mental de muitas pessoas, por restringir o toque, a comunicação próxima e as demonstrações de afeto. Essas medidas podem ser consideradas, muitas vezes, desagradáveis pela boa parte da população, já que se evita o contato direto entre as pessoas, como o toque, o abraço, entre outros. Mudando assim, bruscamente não só as atividades realizadas nos meios de trabalho e convívio social, como também os modos de se relacionar em comunidade. A incerteza do retorno à "vida normal" e o distanciamento tem fomentado sensações de imprevisibilidade, isolamento emocional e até de privação de liberdade (Fundação Oswaldo Cruz, 2020).

Esses sentimentos de incerteza somado ao contexto social e as medidas preventivas de Isolamento Social, acabam por muitas vezes tornando-se catalisadores frequentes para o desencadeamento de reações estressoras, sintomas de ansiedade e até depressão, trazendo à tona sensações de falta de controle emocional (Ramírez-Ortiz et al. 2020).

Concomitante a isso, várias notícias negativas exibidas pela mídia sobre a Covid-19, além das fake news, podem gerar nos indivíduos o estado de alerta constante, correlacionado ao medo de se contaminar e de morrer. Os meios de comunicação muitas vezes usam mensagens de elevação do risco que pode intensificar a ansiedade da população e a angústia (Gualano, Lo Moro, Voglino, Bert, \& Siliquini, 2020).

Frente a essa conjuntura de crises atravessada pela pandemia da Covid-19, as consequências psicológicas e sociais podem acontecerem em variados graus de intensidade e severidade, tornando emergente a Saúde Mental da população, que está enfrentado circunstâncias de perdas e de reorganizações diante do "novo normal" estabelecido para diminuir a propagação da doença, através do distanciamento social, uso de máscaras e cuidados de higienes rigorosos.

A Organização Pan-Americana da Saúde, Unidade de Saúde Mental e Uso de Substâncias (La unidad de Salud Mental y Uso de Sustancias, OPS/OMS), chamam a atenção das autoridades governamentais para os impactos da Covid-19 na saúde mental da população, especialmente em indivíduos que se encontram em condição de exclusão social, idosos e com doenças crônicas, fazendo-se necessário identificar as diferenças entre os grupos populacionais, associando-se ao gênero, idade e condição econômica (Organização Pan-Americana da Saúde, 2016).

Para a Organização Mundial da Saúde (2002), há descobertas que comprovam que o comportamento humano é 
caracterizado, em parte, por suas relações com o meio social e ambiental, de modo que as desordens mentais e comportamentais podem ser avaliadas como má adaptação apreendidas ao longo da vida, afetando assim, em sua saúde mental, sendo essa entendida como habilidades que o sujeito desempenha na comunidade de forma equilibrada e produtiva. É essencial compreender como o contexto da pandemia vem modificando essas relações e afetando as condições de saúde mental das populações, fazendo-se assim, imprescindível pensar sobre as estratégias para o cuidado desses sujeitos. Pensando nessa perspectiva, quais estratégias de cuidado em saúde mental existem? O que a literatura fala sobre estratégias de saúde mental no atual cenário de pandemia? Diante disso, esse trabalho tem como objetivo analisar as estratégias promotoras de saúde mental durante a pandemia pela Covid-19.

\section{Metodologia}

Tendo em vista tal conteúdo para investigação realizou-se como método a Revisão Integrativa (RI) baseada nos pressupostos de Mendes, Silveira \& Galvão (2008), que propõe o estabelecimento de critérios bem definidos sobre a coleta de dados, síntese e a realização de uma análise e apresentação dos resultados, desde o início do estudo, a partir de um protocolo de pesquisa previamente elaborado e validado, a partir das seis etapas indicadas na Figura 1:

Figura 1 - Processo de revisão integrativa.

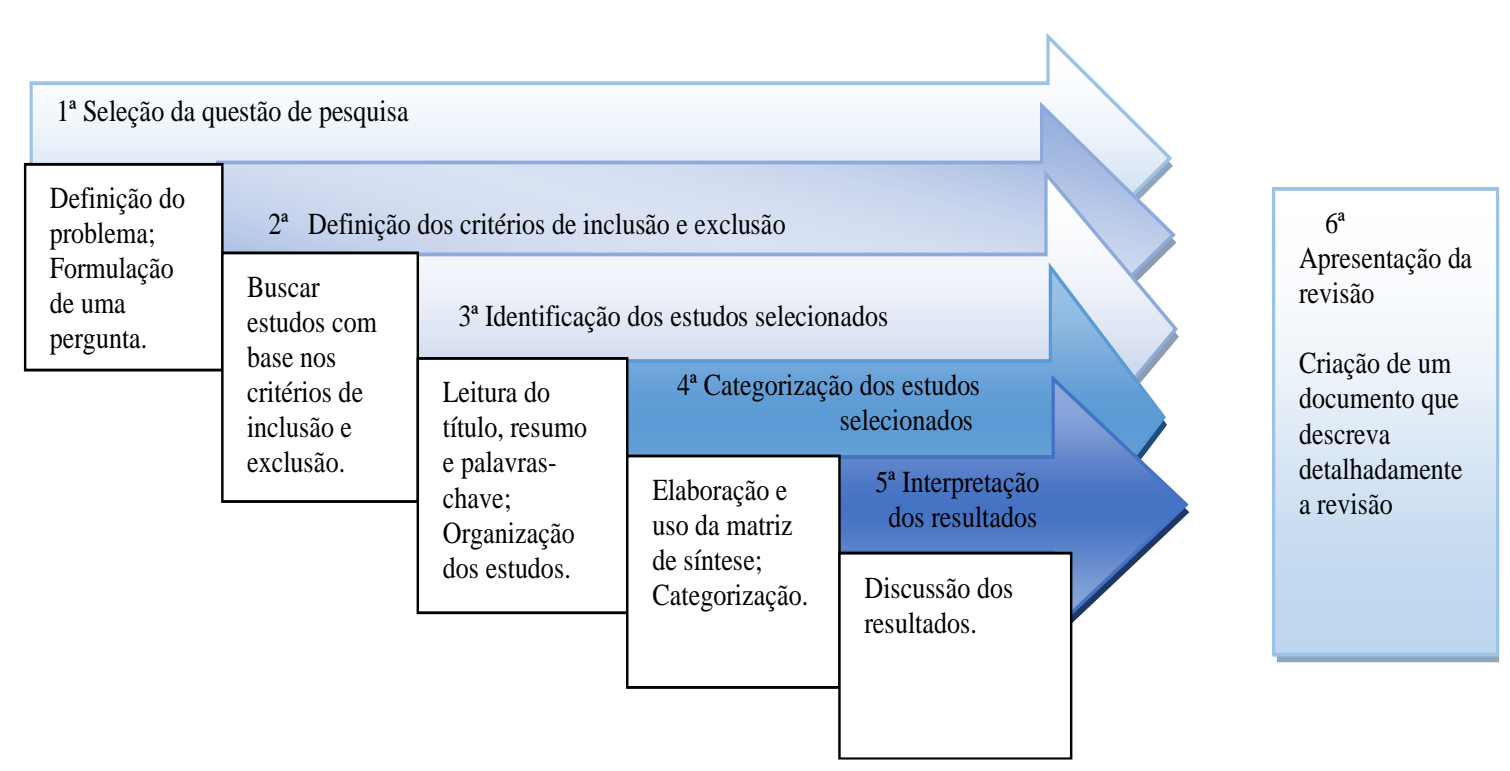

Fonte: Autores (2021).

Para a formulação da questão norteadora do estudo utilizamos a pergunta PCC, a estratégia PICO adaptada. Na adaptação, tais mnemônicos significa P- população, C- conceito e C- contexto/desfecho. Assim, a estratégia foi formulada da seguinte maneira: P- Humanidade; C- Estratégias promotoras de saúde mental; C- pandemia Covid-19 (Peters et al. 2015).

Desta forma estabeleceu-se a seguinte questão: Quais estratégias de cuidado em saúde mental existem? O que a literatura fala sobre estratégias de saúde mental no atual cenário de pandemia? Quais são as estratégias promotoras da saúde mental diante a pandemia da Covid-19?

Após identificação dos Descritores em Ciências da Saúde (DeCS), uma estratégia de busca detalhada, e individual foi realizada na Pubmed, Literatura Latino-Americana e do Caribe em Ciências da Saúde (LILACS) via Biblioteca Virtual em Saúde (BVS). Utilizou-se três descritores extraídos do DeCS “saúde mental, "covid-19", "pandemias” e uma palavra-chave 
"estratégia de saúde mental" a qual reporta significativamente ao objeto de estudo. E para a busca complementar utilizou-se sites de órgãos governamentais e serviços de saúde para levantamento de estratégias de saúde mental (Botelho \& Oliveira, 2017).

Durante a estratégia de busca, foram elencados como critérios de inclusão: artigos completos disponíveis integralmente, no idioma português e/ou inglês e/ou espanhol, artigos referentes ao último ano, informações complementares utilizando-se sites de órgãos governamentais e serviços de saúde; publicações que atendessem a pergunta norteadora. Foram excluídas publicações que preenchiam pelo menos um dos seguintes critérios: (1) revisões; (2) cartas ao editor, (3) opinião pessoal de autores; (4) resumo de encontros; (5) vídeos; (6) ausência de dados relacionados ao objeto e estudo.

Quadro 1 - Equações de buscas nas bases de dados.

\begin{tabular}{cl}
\hline BASE & \multicolumn{1}{c}{ ESTRATÉGIA DE BUSCA } \\
PubMed & $\begin{array}{c}((\text { mental health strategy) AND (covid-19)) AND (pandemic)) AND (mental health) Filters: } \\
\text { Full text, in the last 1 year, Portuguese. }\end{array}$ \\
LILACS via BVS & $\begin{array}{l}\text { (estratégias de saúde mental) AND (covid-19) AND (pandemias) AND (saúde mental) AND ( } \\
\text { db:("LILACS") AND type_of_study:("qualitative_research") AND la:("en" OR "pt" OR "es")) } \\
\text { AND (year_cluster:[2016 TO 2021]) }\end{array}$ \\
$\begin{array}{c}\text { Sites Governamentais \& } \\
\text { Serviços de Saúde }\end{array}$ & Estratégias de saúde mental diante a Covid-19 \\
\hline
\end{tabular}

Fonte: Autores (2021).

A combinação dos termos foi selecionada e adaptada para cada base de dados (Quadro 1). O software Endnotweb foi utilizado para a organização de todas as referências e a remoção dos artigos duplicados. Para a análise dos dados, foi utilizada a Análise de Conteúdo de Bardin (2011).

\section{Resultados}

A busca inicial teve a seguinte distribuição entre as publicações encontradas: PubeMed ( $n=1072)$, BVS ( $n=482)$, Sites de órgãos governamentais e serviços de saúde ( $\mathrm{n}=05$ ), totalizando (1.559) publicações. Em seguida, foram aplicados os filtros, destes foram excluídos $(n=1.507)$ por não se adequarem aos critérios de inclusão da pesquisa. Sobraram $(n=57))$ para serem lidos os títulos e os resumos, foram excluídos $(\mathrm{n}=39)$ por não se adequarem à temática do estudo ou serem artigos de revisão. Assim, restaram $(n=26)$ para serem analisados, contudo destes $(n=16)$ não se encaixaram. As etapas deste processo estão descritas na forma de um fluxograma (Figura 2). 
Figura 2 - Fluxograma da seleção das publicações para a revisão integrativa baseado no modelo PRISMA.

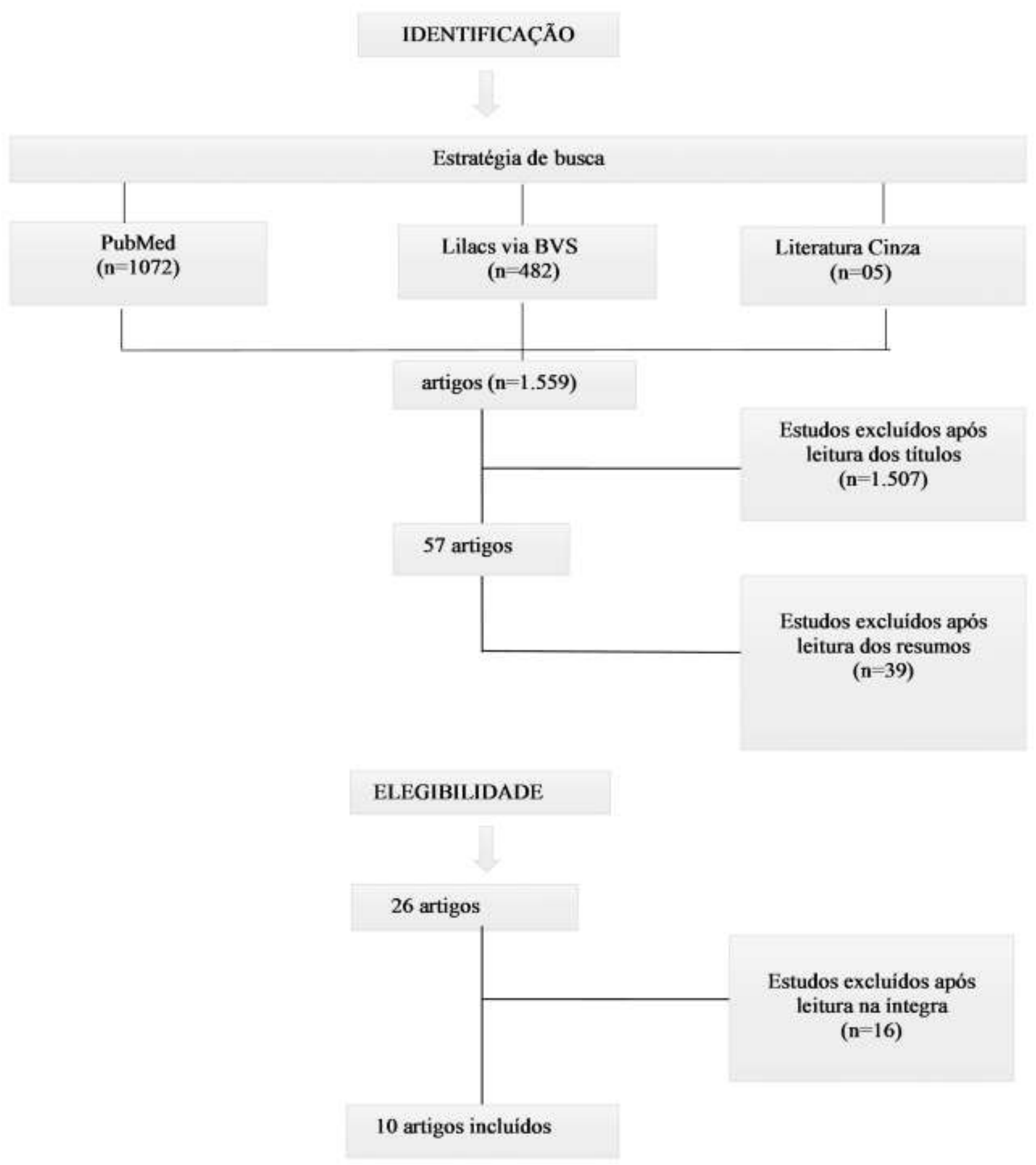

Fonte: Autores (2021).

Sendo assim, restaram $(\mathrm{n}=10)$ para análise e elaboração da matriz de síntese (Quadro 2). As etapas deste processo foram descritas na forma de um fluxograma (Figura 1) adaptado do Reporting Items Systematic and Meta-Analyses (PRISMA). 
Quadro 2 - Caracterização dos estudos incluídos na Revisão Integrativa.

\section{TÍTULO}

Mental health and internet use by

university students: coping strategies in the context of Covid-19 (2021)

Coping strategies, concerns, and habits of Brazilian men in the COVID-19 context

$$
\text { (2021) }
$$

The health of healthcare professionals coping with the Covid-19 pandemic (2020)

\section{AUTOR}

Mota, D. C. B., Silva, Y.

V. D., Costa, T. A. F.,

Aguiar, M., Marques, M.

E. M., \& Monaquezi, R.

Sousa, A. R., Teixeira, J

R. B., Mota, T. N.,

Santana, T. D. S.,

Santos, S. D. D.

Merces, M. C. D., ... Sousa Á, F. L.

Teixeira, C. F. S., Soares, C. M., Souza, E. A., Lisboa, E. S., Pinto, I. C. M., Andrade, L. R., \& Espiridião, M. A

\section{IDIOMA}

Português

Inglês

\section{Ciênc. saúde}

coletiva

\section{Português}

Inglês

Português

Inglês

\section{PRINCIPAIS RESULTADOS}

A população pesquisada apresentou uma prevalência de TMC de 58,5\%, que correlacionou-se positivamente com a frequência de uso de internet, e com a utilização de estratégias de enfrentamento evitativas, baseadas no confronto, orientadas para a busca de suporte social e aceitação de responsabilidade $(\mathrm{p}<0,05)$. A presença de TMC e a utilização de estratégias de enfretamento baseadas no confronto foram as variáveis que melhor explicaram o uso problemático de internet $(\mathrm{R} 2=0,33 \% ; \mathrm{p}<0,00)$. A intensa sociabilidade digital deve ser levada em consideração nas ações de cuidado em saúde mental direcionadas aos universitários no contexto da Covid-19

Como estratégias de enfrentamento predominaram: uso exclusivo do sistema privado de saúde (36,4\%), suporte de familiares/amigos(as) $(78,2 \%)$ e atividades de lazer $(97,7 \%)$ e domésticas $(64,9 \%)$. Distanciamento social $(59,7 \%)$, situações econômica $(58,0 \%)$ e de trabalho $(44,4 \%)$ foram os principais motivos de preocupação. Dentre as atitudes de prevenção/controle prevaleceram a lavagem das mãos $(94,3 \%)$ e o distanciamento social $(91,0 \%)$. Consumo de mídias $(84,6 \%)$ e de risco à saúde $(65,4 \%)$ foram os principais hábitos aumentados.

resgata a análise dos problemas crônicos que afetam os trabalhadores de saúde, decorrentes do sub-financiamento do SUS, do congelamentos dos gastos no setor, da deterioração dos serviços e da precarização da força de trabalho e aponta os desafios agudos que se apresentam à gestão do trabalho e capacitação de pessoal, diante da expansão da infraestrutura de leitos hospitalares e da reorganização do processo de trabalho na atenção básica para o enfrentamento da pandemia, enfatizando as medidas necessárias para a proteção e a promoção da saúde física e mental dos profissionais e trabalhadores da saúde. 
Research, Society and Development, v. 10, n. 11, e199101119368, 2021

(CC BY 4.0) | ISSN 2525-3409 | DOI: http://dx.doi.org/10.33448/rsd-v10i11.19368

Sentimento e emoções de homens no

enquadramento da doença covid-19 (2020)

Fatores associados ao enfrentamento da pandemia da covid-19 por pessoas idosas com comorbidades (2021)

Pandemia e imigração: famílias haitianas no enfrentamento da covid-19 no brasil

Saúde mental das juventudes e covid-19: discursos produtores do webcuidado educativo mediados na webrádio (2020)

A quarentena na covid-19: orientações e estratégias de cuidado (2020)

Saúde mental e atenção psicossocial na covid-19: um guia para gestores (2020)

Como lidar com os aspectos psicossociais e de saúde mental referente ao surto de covid -19 .

\section{Sousa, A. R. d., Carvalho, E. S. d. S., Santana, T. d. S., Sousa, Á. F. L., Figueiredo, T. \\ F. G., Escobar, O. J. V., ... Pereira, Á}

Souza Filho, Z. A. d., Nemer, C. R. B.,

Teixeira, E., Neves, A.

L. M. d., Nascimento,

M. H. M., Medeiros, H.

P., . . Oliveira, V. L. G.

d.

Souza, J. B. d.,

Heidemann, I. T. S. B.

Geremia, D. S.

Madureira, V. S. F.,

Bitencourt, J. V. d. O

V., \& Tombini, L. H. T.

Torres, R. A. M., Veras,

K. d. C. B. B., Torres, J.

D. M., Gomes, E. D. P.,

Martins, J. V., \& Soeiro,

A. C. C.

D.F

Ministério da Saúde

Gagliato, M.

$\begin{array}{cc}\text { Português } & \text { Ciênc. Saúde } \\ \text { Inglês } & \text { Colet. }\end{array}$

Português Esc. Anna.

Inglês Nery

Português

Inglês

$$
\begin{aligned}
& \text { Esc. Anna. } \\
& \text { Nery }
\end{aligned}
$$

BDENF -

Português Enfermagem

Português Fundação

Oswaldo Cruz

Português

Português

Fio Cruz
Prevaleceram sentimentos negativos e ansiedade como consequência do conhecimento acerca do crescente número de hospitalizados e mortos pela pandemia veiculada nos noticiários. Para os homens, o otimismo é necessário para encorajar atitudes com responsabilidade e confiar de que a crise será superada

Quanto às ações de enfrentamento relacionadas a aquisição de informações sobre a COVID-19, observou-se que as pessoas idosas com comorbidades possuem três vezes maior chance (OR=3,07 IC95\% 1,28-7,37) de sempre acompanhar as informações transmitidas pela televisão. $\mathrm{E}$ ainda possuem a maior chance de estarem sempre (OR=2,37 IC95\% 1,19-4,72) e muitas vezes OR=2,34 IC95\% $1,15-4,75)$ satisfeitos ou até mesmo algumas vezes satisfeitos ( $O R=2,18$ IC95\%

$1,14-4,17)$ com as informações que estão sendo veiculadas a respeito do novo coronavírus.

As preocupações geradas no enfrentamento da pandemia pelos participantes foram: trabalho e subsistência da família no Brasil e no Haiti; incerteza do futuro; risco de contaminação e de morrer no Brasil; cancelamento das aulas dos filhos; desânimo e solidão. Desvelaram oportunidades na vivência da pandemia: ajuda recebida; força pessoal e familiar; repensar a vida; confiança em Deus e esperança.

A comunicação produzida na webrádio ajudou os jovens escolares e universitários no desenvolvimento de estratégias de aproximação, escuta e interações no contexto do isolamento social, auxiliando- os nas tomadas de decisões para prevenção e contenção da pandemia da Covid-19.

Cartilha de orientações e estratégias de cuidado frente a quarentena na Covid-19

Guia de orientação aos gestores

OPAS
Guia preliminar

Fonte: Autores (2021). 


\section{Discussão}

Ao discutir sobre pandemia de Covid-19 e Saúde Mental é fundamental levar em consideração o contexto social que a pessoa está inserida para que assim seja possível identificar os fatores e desse modo serem criadas estratégias de enfrentamento, já que o observado é que apenas o isolamento social e o distanciamento por si só não são fatores desencadeantes de adoecimento mental, como retrata Sousa et al (2021). As questões sociais relacionadas ao crescente desemprego, precariedade do trabalho, diminuição da renda familiar, restrição das atividades compartilhadas em comunidade e a perda familiar tem se mostrado como os fatores mais alarmantes e contribuidores para o sofrimento mental no contexto atual.

Desse modo, o que tem se encontrado no Brasil, como forma de estratégias para lidar com esse crescente sofrimento mental é a disponibilização de psicólogos para prestar auxílio e acolhimento àqueles que têm sido psicologicamente afetados pela pandemia da Covid-19. O governo tem convocado profissionais de saúde para prestar trabalho voluntário para escutar esse público que encontra-se em sofrimento, além do trabalho voluntário, muitos profissionais da saúde foram contratados em serviço de tempo delimitado para atender a essas demandas específicas (Ministério da Saúde, 2020b).

Psicólogos e psiquiatras, para darem continuidade aos cuidados em saúde mental durante a pandemia, estão se mobilizando para realizar intervenções e atendimentos online. Medidas como essas podem ajudar a diminuir ou prevenir futuros problemas psiquiátricos e psicológicos (Cullen, Gulati, \& Kelly, 2020; Lima et al., 2020; Wind, Rijkeboer, Anderson, \& Riper, 2020,). Os atendimentos online foram uma forma de dar conta não só das questões mais urgentes, como também uma forma de prevenir a saúde mental da população, nela incluída os próprios profissionais de saúde que atuam na linha de frente ao combate a Covid-19.

Percebeu-se que no primeiro momento os olhares foram voltados para a prestação de cuidados físicos e todas as medidas de segurança que poderiam ser tomadas para a diminuição da propagação do vírus, até porque a doença espalhou-se rapidamente e teve agravos severos na sociedade, porém o que foi percebendo-se é que a Covid-19 não se instalou apenas como uma onda ou doença passageira, de modo que foi preciso repensar os cuidados que estavam sendo reproduzidos, passando a incorporar o cuidado em saúde mental como algo primordial ao falar-se da doença.

Para além dos cuidados prestados a pacientes com a Covid-19 é importante pensar na necessidade de manutenção de um acesso equitativo à prestação de serviços essenciais durante uma emergência, reduzindo assim o número de mortalidade direta e indireta. Este dado ressalta a necessidade de uma organização que possibilite o funcionamento dos serviços essenciais à saúde, garantindo força de trabalho para lidar com necessidades para além da Covid-19. Fato esse que podemos relacionar como uma estratégia de promoção de saúde, incluindo-se aqui a saúde mental, pois dentre os serviços essenciais a OMS destacou àqueles que garantem o calendário de vacinação, os cuidados durante a gravidez e parto, o tratamento de doenças infecciosas e não transmissíveis, os serviços de sangue e os dispositivos da rede de saúde mental, o que, na realidade brasileira, refere-se aos pontos da Rede de Atenção Psicossocial (RAPS) no âmbito do Sistema Único de Saúde (SUS) (Barbosa et al. 2020, p. 11). Sendo assim, os dispositivos da rede de saúde mental precisam estar atuantes para garantir assim, o cuidado em saúde mental, mesmo que as demandas não estejam atreladas essencialmente as repercussões da Covid-19.

Evidencia-se também a relevância de medidas que assegurem o cuidado a grupos vulneráveis, como os idosos, refugiados, crianças, entre outros, sustentando o bem-estar dessas pessoas que perderam sua renda e possuem necessidades de assistência básica e cuidados essenciais, de modo a garantir apoio e fomento à resiliência e à saúde mental (Barbosa et al, 2020).

No tangente aos idosos, faz-se imprescindível ter prudência não só com as questões fisiológicas em consequência do envelhecimento, mas também no que concerne às alterações emocionais e sociais desses indivíduos, que durante a pandemia podem ter agravado situações de dependência e diminuição de suas capacidades funcionais, de modo a reverberar 
significativamente no modo de vida dessas pessoas e na forma como elas experienciam sua singularidade (Souza Filho et al. 2021).

Diferente de outros grupos, o isolamento para os idosos tornou-se algo inquestionável, devido a eles serem a população de maior risco de agravo/mortalidade no que se refere a Covid-19. Frente a esse dado, o medo de complicações e da morte se tornou algo intrínseco à vivência do ser idoso no contexto atual. Para tanto, é possível pensar em estratégias que visem experienciar o envelhecimento nesse cenário contemporâneo, aproveitando-se das tecnologias como aliadas nesse processo, já que a inclusão digital pode favorecer o processamento cognitivo em idosos, como também aproximá-los do contato com familiares e amigos em tempo real e até mesmo dar conta de atividades externas que são disponibilizadas por serviços de entregas (Souza Filho et al, 2021).

Outra estratégia interessante, quando se trata de grupos específicos da população, é identificar primeiramente as vulnerabilidades daquele determinado grupo, como é o caso dos homens, uma população que tem chamado a atenção quando o assunto é a morbimortalidade em relação a Covid-19. Estudos mostram que há conformidades que podem ser explicadas através dos fatores genéticos, comportamentais e de estilo de vida dessa população, trazendo assim incitações dos processos da masculinidade que pertencem ao enquadramento sócio-histórico da doença, como a negação da gravidade da doença, o descumprimento das medidas sanitárias, a baixa procura por serviços de saúde na Atenção Primária e até mesmo a presença de narrativas voltadas para o pessoal, tornando esse homem um pouco distante dessa ideia do coletivo (Sousa et al. 2020).

Destarte vale-se a reflexão sobre esse público específico de modo a buscar considerações que alcancem questões relacionadas à masculinidade, como o reconhecimento da própria vulnerabilidade, consciência coletiva sanitária e modificação da cultura referente aos cuidados em saúde mental, à procura de garantir resolubilidade e coerência para essas singularidades (Sousa et al. 2020).

Um estudo transversal, realizado através de ambiente virtual, com homens residentes no Brasil retrata que grande parte dos homens adotou algum tipo de estratégia para enfrentamento da pandemia $(97,9 \%)$ e as que mais predominaram foram as atividades de lazer (97,7\%) e doméstica (64,9\%) (Souza et al. 2021).

No referente à população geral, a promoção à saúde mental e o objetivo de prevenir quanto a consequências negativas, tem se orientado adotar estratégias de enfrentamento, que permita uma adequação à realidade do distanciamento social. Tais orientações podem potencializar a produção de equilíbrio psicológico de forma autônoma, podendo evitar, em grande parte dos casos, que haja necessidade de cuidados especializados (Fundação Oswaldo Cruz, 2020).

Alguns guias de como enfrentar os aspectos Psicossociais e de Saúde Mental diante a pandemia pela Covid-19 foram criados, objetivando orientações, a partir de site de saúde e governamentais enfatizando a importância de saber lidar com aspectos psicossociais e da saúde mental, frente a Covid-19 (Gagliato, 2020).

Tais guias trazem alguns pontos em comum, dentre eles: o entendimento sobre saúde mental, o contexto de emergência comunitária, assim como questões enfatizando o estresse e a preocupação, em trabalhadores da linha de frente, a estigmatização e as vulnerabilidades sociais na população em geral. No que diz respeito ao contexto de atenção em saúde mental, tais como, a rede de atenção psicossocial, é colocado a necessidade de estruturas locais de condução e apoio, ambientes protetivos, tratamentos para sociedade em geral e estratégias a curto, médio, longo prazo.

Além disso, a OMS vem publicando diversas mensagens de apoio ao bem-estar mental e psicossocial. Elas são encaminhadas a vários grupos que compõe a sociedade, recomendando que a população busque informações confiáveis sobre a Covid-19 (Ministério da Saúde, 2020b). 


\section{Considerações Finais}

Esse artigo reuniu conhecimento acerca da saúde mental e dos aspectos associados à emergência de saúde pública por conta do novo coronavírus. Foram expostas questões relacionadas a problemas do campo de saúde mental, fatores protetivos no desenvolvimento de transtornos mentais e, também, exemplos de possíveis estratégias em diferentes momentos da pandemia.

Foi possível apreender nesse estudo que a intervenção e o manejo de crise foram uteis para diminuir a curva de contágio, embora o número de infectados tenha sido altíssimo e as medidas de contenção para a diminuição da disseminação da Covid-19 precisem continuar. Percebe-se que as ações essenciais que não estão ligadas diretamente a Covid-19 precisam ser recolocadas em foco, valorizando o quanto essas ações podem também contribuir para a saúde mental da população.

O atual cenário de potencial catástrofe em saúde mental exige esforços que devem ser empregados, em todos os níveis e pelas mais diversas áreas de conhecimento, a fim de minimizar resultados ainda mais negativos na saúde mental da população. Cabe salientar, que este trabalho não abarca, obviamente, todo o alcance da produção científica em relação as estratégias de enfrentamento da pandemia pela Covid-19, levando em consideração que ainda estamos atravessando esse período pandêmico.

Percebe-se a necessidade de mais estudos que abordem as estratégias de promoção da saúde mental visto que o adoecimento mental é inevitável no contexto da pandemia da Covid-19, e as estratégias já encontradas não são necessariamente e integralmente aplicáveis em todos os contextos. Dessa forma, este presente trabalho visa incentivar e potencializar novas discussões sobre a temática e reflexões sobre novas estratégias a curto, médio e longo prazo.

\section{Referências}

Barbosa, A. S, Nascimento, C. V, Dias, L. B. S, Espírito Santo, T. B, Chaves, R. C. S., \& Fernandes, T. C. (2020). Processo de trabalho e cuidado em saúde mental no Centro de Atenção Psicossocial da UERJ na pandemia de COVID-19. Brazilian Journal of Health and Biomedical Sciences, 19(1), 11-19. https://doi.org/10.12957/bjhbs.2020.53527

Bardin, L. (2011). Análise de conteúdo. Edições 70.

Botelho, L. L. R., Cunha, C. C. A. \& Macedo, M. (2011). O método da revisão integrativa nos estudos organizacionais. Gestão e Sociedade. 5(11), 12136. https://doi.org/10.21171/ges.v5i11.1220.

Cullen, W, Gulati, G. \& Kelly, B. D. (2020). Mental health in the Covid-19 pandemic. QJM: An International Journal of Medicine, 113(5), 311-312. https://doi.org/10.1093/qjmed/hcaa110.

EndNote Web (2021). [place unknown], https://access.clarivate.com/login?app=endnote

Fundação Oswaldo Cruz. (2020). A Quarentena na Covid-19: Orientações e Estratégias de Cuidado. Autor. https://www.fiocruzbrasilia.fiocruz.br/aquarentena-na-covid-19-orientacoes-e-estrategias-de-cuidado/\#: :text=A\%20nov a\%20cartilha\%20aborda\%20orientações,m ental\%20daqueles\%20que\% 20as\%20experienciam.

Gagliato, M. (2020). Guia Preliminar como lidar com os aspectos Psicossociais e de Saúde mental referente ao surto de COVID - 19. Versão 1.5. Brasília, D. F. OPAS. https://www.paho.org/pt/documents/interim-briefing-note-addressing-mental-health-and-psychosocial-aspects-covid-19-outbreak

Gualano, M.R, Lo Moro, G, Voglino, G, Bert, F. \& Siliquini, R. (2020). Effects of Covid-19 Lockdown on Mental Health and Sleep Disturbances in Italy. Int. J. Environ. Res. Public Health, 17(13), 1-11. https://doi.org/10.3390/ijerph17134779

Lima, C. K. T, Carvalho, P. M. M, Lima, I. A. S, Nunes, J. A. V. O, Saraiva, J. S, Souza, R. I. \& Rolim Neto, M. L. (2020). The emotional impact of coronavirus 2019-Ncov (new Coronavirus Disease). Psychiatry Research, 287(1), 1-9. https://doi.org/10.1016/j.psychres.2020.112915

Mendes, K. D. S., Silveira, R. C. P. S. \& Galvão, C. M. (2008). Revisão Integrativa: método de pesquisa para a incorporação de evidências na saúde e na enfermagem. Texto Contexto Enfer., 17(4).

Ministério da cidadania. (2020). Portaria $n^{o}$ 340, de 30 de março de 2020: estabelece medidas para o enfrentamento da emergência em saúde pública de importância nacional decorrente de infecção humana pelo novo coronavírus (covid-19), no âmbito das comunidades terapêuticas. | edição: 62 | seção: 1 | página: 5 https://www.in.gov.br/en/web/dou/-/portaria-n-340-de-30-de-marco-de-2020-250405535

Ministério da Saúde do Brasil. (2020a). Guia de Vigilância Epidemiológica: Emergência de Saúde Pública de Importância Nacional pela Doença pelo Coronavírus 2019. Brasília, D. F.: Fiocruz. Recuperado de https://portaldeboaspraticas.iff.fiocruz.br/biblioteca/guia-de-vigilancia-epidemiologica-emergenciade-saude-publica-de-importancia-nacional/. 
Ministério da Saúde do Brasil. (2020b). Saúde mental e atenção psicossocial na Covid-19: um guia para gestores. Brasília, D. F.: Fiocruz. http://www.fiocruzbrasilia.fiocruz.br/wp-content/uploads/2020/04/Sa\%c3\%bade-Mental-eAten\%c3\%a7\%c3\%a3o-Psicossocial-na-Pandemia-Covid-19recomenda\%c3\%a7\%c3\%b5es-para-gestores.pdf.

Mota, D. C. B., Silva, Y. V. D., Costa, T. A. F., Aguiar, M., Marques, M. E. M., \& Monaquezi, R. M. (2021). Mental health and internet use by university students: coping strategies in the context of COVID-19. Cien Saude Colet, 26(6), 2159-2170. https://doi.org/10.1590/1413-81232021266.44142020 (Saúde mental e uso de internet por estudantes universitários: estratégias de enfrentamento no contexto da Covid-19.)

Organização Pan-Americana da Saúde. (2016). Protección de la salud mental y atención psicosocial en situaciones de epidemias. Brasília, D. F.: OPAS. https://www.paho.org/disasters/index.php?option=com_docman\&view=download\&category_slug=informes-tecnicos\&alias=2539-proteccion-salud-mentalatencion-psicosocial-situaciones-epidemias-2016-539\&Itemid=1179\&lang=en

World Health Organization. (2002). Mental Health: New Understanding, New Hope. https://www.who.int/whr/2001/en/whr01_djmessage_po.pdf

Peters M. D. J., Godfrey C. M, Khalil H., McInerney P., Parker D. \& Soares C.B. (2015) Guidance for conducting systematic scoping reviews. Int J Evid Based Healthc [Internet]. (3): 141-6. https://www.researchgate.net/publication/319713049_2017_Guidance_for_the_Conduct_of_JB I_Scoping_Reviews?enrichId=rgreq-2c63bf47a03bf1c379fed09bf9a175b4-XXX\&enrichSource=Y292ZXJQYWd1OzMxOTcxMzA0OTtBUzo1NDA5MDcx MjY4ODY0MDBAMTUwNTk3MzcxNjg4MA\%3D\%3D\&el=1_x_2\&.

Sousa, A. R., Teixeira, J. R. B., Mota, T. N., Santana, T. D. S., Santos, S. D. D., Merces, M. C. D., \& Sousa Á, F. L. (2021). Coping strategies, concerns, and habits of Brazilian men in the Covid-19 context. Rev Bras Enferm, 74Suppl 1(Suppl 1), e20210040. https://doi.org/10.1590/0034-7167-2021-0040

Sousa, A. R. d., Carvalho, E. S. d. S., Santana, T. d. S., Sousa, Á. F. L., Figueiredo, T. F. G., Escobar, O. J. V., \& Pereira, Á. (2020). Sentimento e emoções de homens no enquadramento da doença Covid-19 Ciênc. Saúde Colet, 25(9), 3481-3491.

Souza Filho, Z. A. d., Nemer, C. R. B., Teixeira, E., Neves, A. L. M. d., Nascimento, M. H. M., Medeiros, H. P., \& Oliveira, V. L. G. d. (2021). Fatores associados ao enfrentamento da pandemia da Covid-19 por pessoas idosas com comorbidades. [Esc. Anna Nery Rev. Enferm, 25(spe), e20200495-e20200495.

Souza, J. B. d., Heidemann, I. T. S. B., Geremia, D. S., Madureira, V. S. F., Bitencourt, J. V. d. O. V., \& Tombini, L. H. T. (2020). Pandemia e imigração: famílias haitianas no enfrentamento da Covid-19 no Brasil. Esc. Anna Nery Rev. Enferm, 24(spe), :e20200242-:e20200242.

Teixeira, C. F. S., Soares, C. M., Souza, E. A., Lisboa, E. S., Pinto, I. C. M., Andrade, L. R., \& Espiridião, M. A. (2020). The health of healthcare professionals coping with the Covid-19 pandemic. Cien Saude Colet, 25(9), 3465-3474. https://doi.org/10.1590/1413-81232020259.19562020 (A saúde dos profissionais de saúde no enfrentamento da pandemia de Covid-19.)

Torres, R. A. M., Veras, K. d. C. B. B., Torres, J. D. M., Gomes, E. D. P., Martins, J. V., \& Soeiro, A. C. C. (2020). Saúde mental das juventudes e Covid-19: discursos produtores do webcuidado educativo mediados na webrádio.Nursing (Säo Paulo), 23(270), 4887-4896.

Ramírez-Ortiz, J, Castro-Quintero, D, Lerma-Córdoba, C, Yela-Ceballos, F. \& Escobar-Córdoba, F. (2020). Consecuencias de la pandemia Covid-19 en la Salud Mental asociadas al aislamiento social. Colombian Journal of Anestesiology, 48(4), 1-8. https://doi.org/10.5554/22562087.e930

Wind, T.R, Rijkeboer, M, Andersson, G. \& Riper, H. (2020). The Covid-19 pandemic: the 'black swan' for mental health care and a turning point for e-health. Internet Interventions, 20(1), 1-9. http://dx.doi.org/10.1016/j.invent.2020.100317. 